This is a postprint version of the following published document: L. Ruiz; I. González; R.J. Durán; I. de Miguel; N. Merayo; J.C. Aguado; P. Fernández; R.M. Lorenzo; E.J. Abril. Comparing Different Types of Flexibility when Solving the RSA Problem in EONs. In: 2017 International Conference on Computational Science and Computational Intelligence (CSCI). DOI: https://doi.org/10.1109/CSCI.2017.236

(C) 2017 IEEE. Personal use of this material is permitted. Permission from IEEE must be obtained for all other uses, in any current or future media, including reprinting/republishing this material for advertising or promotional purposes, creating new collective works, for resale or redistribution to servers or lists, or reuse of any copyrighted component of this work in other works. 


\title{
Comparing Different Types of Flexibility when Solving the RSA Problem in EONs
}

\author{
L. Ruiz, I. González, R.J. Durán, I. de Miguel, N. Merayo, J.C. Aguado, P. Fernández, R.M. Lorenzo and E.J. Abril \\ Universidad de Valladolid \\ Valladolid (Spain) \\ \{ 1ruiper@ribera.tel.uva.es, rduran@tel.uva.es
}

\begin{abstract}
Elastic Optical Networks (EON) are seen as an essential technology to implement the backhaul of Future Internet allowing the deployment of emerging paradigms like the Internet of Things (IoT), Tactile Internet, or Industry 4.0. When designing those Elastic Optical Networks (EONs), the Routing and Spectrum Assignment (RSA) problem has to be solved. In this paper, we analyse the effect of using two types of flexibility in a well-known RSA method. A simulation study will be presented with the main results of this comparison.
\end{abstract}

Keywords-Elastic Optical Networks, Routing and Spectrum Assignment, Flexibility, IoT

\section{Full Research Paper-CSCI-ISOT}

\section{INTRODUCTION}

The future (and present) explosion of emerging paradigms like the Internet of Things (IoT), Tactile Internet or Industry 4.0 are possible due to the evolution of communication infrastructures. Those new services impose stringent requirements that current networks cannot offer [1]. Fibre networks are the best choice to deploy backhaul infrastructures, besides offering high bandwidth, they provide dynamicity, scalability and reliability [2].

Wavelength-Routed Optical Networks (WRON) use Wavelength Division Multiplexing (WDM) in order to establish optical circuits or lightpaths between network nodes (not necessarily adjacent in the physical network) [3]. Lightpaths can be established in the network in two different ways: semi-static (also static) or dynamic. In the former paradigm, a set of lightpaths are established in the network, depending on the expected or measured traffic, forming the virtual topology. On the contrary, in dynamic networks, the requests for establishment of lightpaths arrive in real time. In these dynamic networks, the problem is to find a route and a wavelength channel to establish the lightpath. This problem is usually known as Dynamic Routing and Wavelength Assignment problem (RWA).

Current WRONs use ITU-T fixed channels in order to establish the lightpaths but its utilization does not allow

This work has been supported by Spanish Ministry of Economy and Competitiveness (TEC2014-53071-C3-2-P, TEC2015-71932-REDT) and the fellowship program of the Spanish Ministry of Education, Culture and Sports (BES-2015-074514) accommodating traffic in the most effective way, causing a waste of network capacity (and efficiency). In order to solve this problem, a first step consists in the migration of current single line rate WRONs towards mixed line rate solutions, where channels with different line rates (e.g., 10, 40 and 100 Gbps) coexist in the same network but using the ITU-T defined channels. However, in order to improve network efficiency, flexible optical network architectures, such as SLICE [4], were proposed. These Elastic (or flexible) Optical Networks (EONs) allow the allocation of a variable portion of spectrum to each optical connection according to its requirements. That can be done by using techniques like OFDM or Nyquist-WDM [5,6], for which that traffic is transported on several low data rate subcarriers, whose number and/or modulation format is adapted according to the traffic demand, thus providing subwavelength granularity for low rate demands or super-channels for high rate ones. The adoption of EONs based on reconfigurable bandwidth-variable transponders and bandwidth-variable wavelength cross-connects, with strong constraints in terms of network flexibility to rapidly provision optical bandwidth will provide suitable support the future demand of $5 \mathrm{G}$ and IoT applications.

In future EONs, the RWA problem is transformed into the Routing and Spectrum Allocation (RSA) problem [7]. In RSA, a portion of the spectrum should be reserved in a sequence of fibers leading from the source to the destination node, providing the capacity required by the user or the network operator. There are different methods and studies to solve the dynamic and static RSA problem in the literature [7-10]. Most of them use narrow spectrum slots allowing the establishment of the lightpath with the desired capacity building a superchannel over a set of contiguous slots. Other methods use defragmentation techniques $[8,11]$ to perform a replacement in the allocated spectrum in order to improve network efficiency. Moreover, the RSA method can be centralized (i.e., solved in a Path Computation Element, PCE) or distributed.

In this paper, we show a comparative study between four different RSA centralized alternatives to allocate capacity for dynamic lightpaths when no defragmentation techniques are used. The four alternatives or algorithms are based on the wellknown method of using $k$-shortest-paths together with the firstfit algorithm to solve the RWA problem in classic WRONs [12]. We modify that algorithm by implementing different types of flexibility. Firstly, we consider the effect of using 
spectrum slots ("Fixed-Grid") or not ("Gridless"). Secondly, we analyze the effect of using a compact spectrum allocation ("Joint spectrum") for each lightpath demand (i.e., building a single superchannel) or allowing using more than one superchannel to complete the bandwidth required by the lightpath request (“Disjoint spectrum”).

\section{RSA AlgorithMS FOR THE DYNAMIC PROBLEM}

The problem of establishing a dynamic lightpath is defined in flexible optical networks with centralized control in a similar manner as in classic WRONs. Lightpath establishment requests arrive at the PCE on real time during network operation, and they are processed, so that if there are enough idle resources during a certain period of time the lightpath is established, and is rejected otherwise [7]. Each lightpath is characterized by its source and destination nodes, and by the spectrum capacity that it requires. If no waveband conversion is used, the same portion of spectrum should be reserved in all the fibers of the route.

As commented before, we will analyze the impact of using different types of flexibility in a well-known RSA method. In "fixed-grid" methods, the spectrum is divided into frequency slots, i.e., narrow spectrum segments of a given width in $\mathrm{GHz}$, all of them with the same bandwidth. When a traffic demand arrives, the algorithm will assign as many available slots as needed to allocate all the data in the traffic demand. In contrast, in "gridless" method, the spectrum is not divided and lightpaths are established using exactly the bandwidth required by the user. It is important to note that in both fixed-grid and gridless methods, a guard band is reserved between two consecutive demands in the spectrum to avoid interference between them.

On the other hand, "joint spectrum" methods assign all the capacity required by the user in one single (and compact) superchannel. In contrast, "disjoint spectrum" methods allow splitting the required capacity into several "sub-lightpaths" with different bandwidths (if necessary to improve the performance of the network) and using traffic grooming mechanisms in the edges of the lightpaths to divide and merge the demanded capacity of the lightpath request.

Therefore, combining these two types of flexibility, we have implemented four different RSA algorithms: Joint Spectrum Fixed Grid (JSF), Disjoint Spectrum Fixed Grid (DSF), Joint Spectrum Gridless (JSG) and Disjoint Spectrum Gridless (DSG). All of them solve independently the routing problem and the spectrum assignment problem. For routing, the $k$-shortest path method is used. For spectrum assignment, the first-fit heuristic [12] is employed. We selected these heuristics as they are very well-known, but the two degrees of flexibility mentioned above could also be used in combination with many other RSA techniques. We assume that waveband conversion is not used.

In JSF, when a traffic request between a source node, $s$, and a destination node, $d$, arrives at the PCE, it checks the precomputed path list between $s$ and $d$ from the shortest to the longest one, divides the spectrum of the fibers in the path in slots of a given granularity and assigns as many free contiguous slots as needed to allocate the demanded bandwidth in the first set of idle and contiguous slot with the capacity of the lightpath request. When JSF finds enough idle spectrum to establish the lightpath, no more routes nor spectrum are analysed. JSG works in a similar way as JSF with the difference of not considering a grid spectrum. Both JSF and JSG implies that only one guard band will be added per lightpath.

DSF uses "fixed-grid" and it allows to divide the requested capacity into (non-contiguous in the spectrum) "sub-lightpaths" and use traffic grooming to deliver the full capacity of the lightpath request. However, a restriction is imposed for simplicity: all the sub-lightpaths have to follow the same path from $s$ to $d$. When DSF searches for the requested capacity in a route, it goes through the spectrum (from lowest to highest frequency) and assigns any idle contiguous spectrum found (only if the idle contiguous spectrum is higher than the guard band) until the demanded capacity of the lightpath request is completed. As the bandwidth of a superchannel has to be compact, more than one superchannel can be established, each one forming a sub-lightpath. DSG works similarly but without dividing the spectrum into slots. In both DSF and DSG, a guard band is added to each sub-lightpath established.

\section{Simulation RESUltS}

\section{A. Simulation scenario}

In order to evaluate the performance of the algorithms, we have implemented a simulator of flexible optical networks using OMNeT++ [13]. The physical network topology used for this study is the 14-node NSFNet, where a cable between two network nodes is assumed to consist of two unidirectional fibers (one for each direction). The available capacity in each fiber is $4000 \mathrm{GHz}$. In those algorithms that divide the spectrum in fix-grid bands, four different size of bands are used: $12.5,25,50$ and $100 \mathrm{GHz}$. The band size of $12.5 \mathrm{GHz}$ is the most used in previous studies of Flexible Optical Networks. The guard band width is $10 \mathrm{GHz}$ (in fixed-grid) this guard band is accommodated inside the last slot used by the superchannel).

Lightpath requests arrive following a Poisson process and the source-destination nodes pairs of each request are randomly selected using a uniform distribution. The demanded bandwidth of each lightpath is selected randomly following a uniform distribution between $1 \mathrm{GHz}$ and $300 \mathrm{GHz}$, and the holding time of a connection is obtained by means of an exponential distribution. All the results are shown with $95 \%$ confidence intervals (although in most cases they are smaller than the size of the symbols).

\section{B. Simulation results}

We have performed simulations with different values of $k$ and, obviously, obtained that the blocking probability with all methods improves as $k$ is higher at the expense of increasing the computing time. In all the following figures, we only show the results with $k=5$ but similar behavior was obtained with other $k$ values.

Fig. 1 shows the blocking probability of the JSF algorithm for the different slot widths. Fig. 2 shows the corresponding values using DSF. 


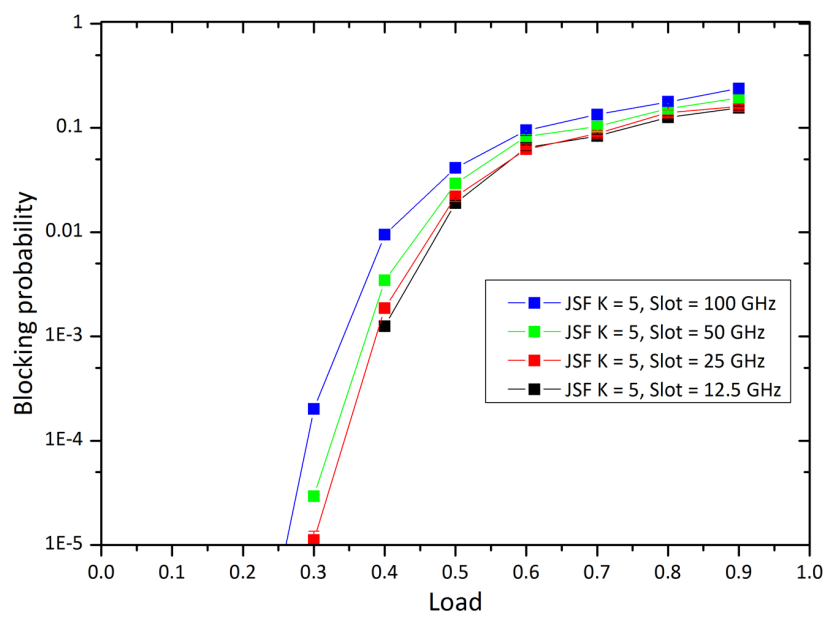

Fig. 1. Blocking probability for JSF with $k=5$ and spectrum granularity of $12.5 \mathrm{GHz}, 25 \mathrm{GHz}, 50 \mathrm{GHz}$ and $100 \mathrm{GHz}$

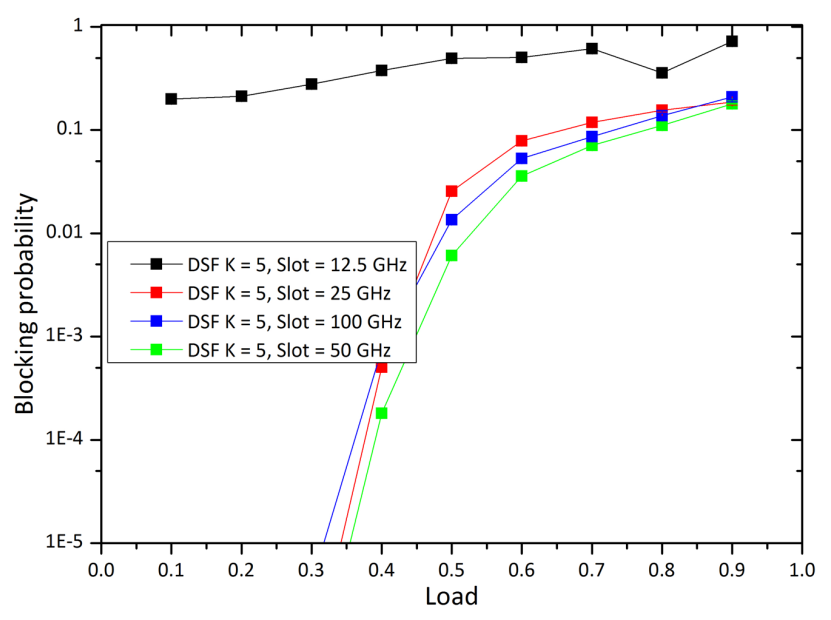

Fig. 2. Blocking probability for DSF with $k=5$ and spectrum granularity of $12.5 \mathrm{GHz}, 25 \mathrm{GHz}, 50 \mathrm{GHz}$ and $100 \mathrm{GHz}$

Fig. 1 shows that when JSF is used, the lowest (and best) blocking probability is achieved when the slot size is 12.5 $\mathrm{GHz}$, although the difference is almost negligible for sizes $12.5,25$ and $50 \mathrm{GHz}$ for high traffic loads. This behavior happens because the lower the slot size is, the least spectrum is wasted. On the contrary, results from Fig. 2 show that the highest blocking probability is obtained using DSF with slot size $12.5 \mathrm{GHz}$.

The problem of DSF when using very narrow slots is that it can split the required capacity into many sub-lightpaths and each one of this sub-lightpaths requires a guard band. Therefore, a lot of spectrum is wasted in guard bands and the network losses capacity and efficiency. The optimal performance with DSF is obtained with a slot size of $50 \mathrm{GHz}$ (see Fig. 2). These results suggest a trade-off between slot size and band guards: having narrow slots increases the precision of the bandwidth allocation but in DSF implies adding a higher number of band guards and, therefore a worse usage of the spectrum.

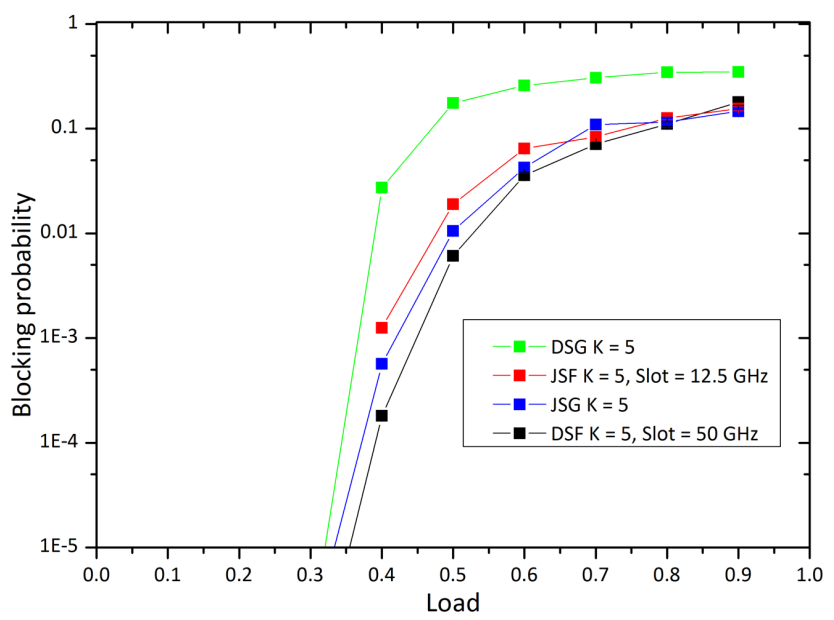

Fig. 3. Blocking probability for the four RSA algorithms with $k=5$

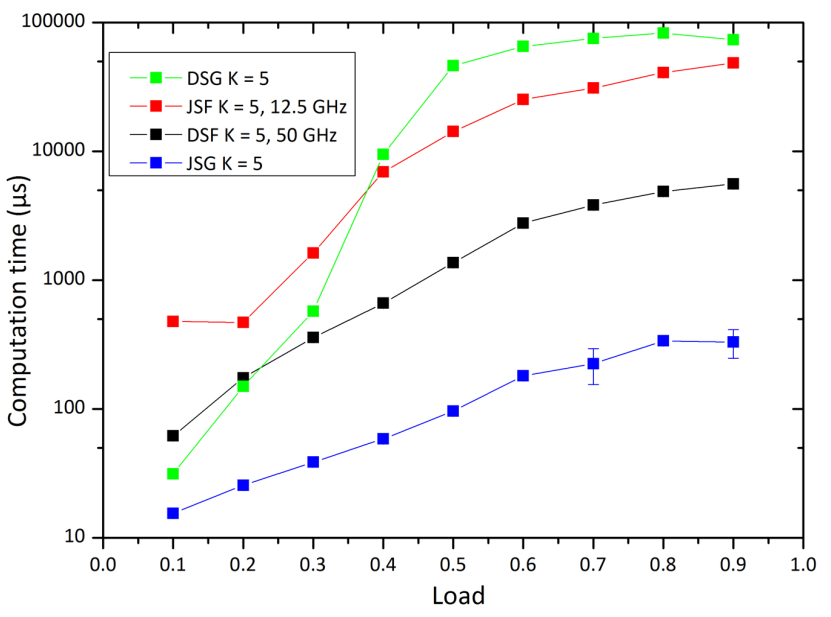

Fig. 4. Computation time for the four algorithms with $k=5$

Fig. 3 shows a comparison in terms of blocking probability for the best performing configuration of each algorithm and we now include the result for the gridless scenario (i.e., JSG and DSG algorithms). A comparison of the computing time that each algorithm requires to find the solution to the RSA problem are shown in Fig. 4.

Fig. 3 shows that the lowest blocking probability is achieved using DSF with slot size of $50 \mathrm{GHz}$. Therefore, the flexibility of using the disjoint spectrum technique can lead to significant improvements in blocking probability. However, this is not the case when it is combined with very narrow fixgrid slots (as shown in Fig. 2) or when used in a gridless scenario (DSG). In fact, Fig. 3 shows that the latter option, DSG, as it allows the splitting of the capacity of a lightpath into many sub-lightpaths (each one requiring a guard band), it wastes a significant amount of spectrum. This effect can be probably solved with spectrum assignment techniques different from First-Fit, but it is still under research. Moreover, it is also important to remark that the use of disjoint methods can provide best performance (e.g. DSF) in terms of blocking probability but increases control complexity and requires more 
resources (the number of transmitters and receivers will be increased, as it needs one per sub-lightpath). Therefore, they lead to an increment in the capital and operating expenses of the network.

On the other hand, with the joint spectrum technique, the use of gridless instead of fixed-grid can also bring benefits as the spectrum of the fiber is used in a more efficient way (as shown in Fig. 3, JSG performs better than JSF).

The results from Fig. 4 show that JSG is the best method in terms of computation time due to the "simplicity" of the operations it performs. DSF is the following best. However, the computational time of JSF and DSG is very high (especially in higher network loads) and, therefore, they cannot be suitable to use them in a dynamic scenario. Note that JSF can reduce its computational time by using bigger slot size at the expense of increasing the blocking probability (Fig. 1)

\section{CONCLUSION}

In this paper, we have presented a performance comparison when considering two types of flexibility to the simple $k$-shortest-paths and first-fit RSA algorithms in terms of the blocking probability and computational time. The results show that the performance of the RSA method can be improved by using disjoint techniques together with not-too-fine slot sizes (in order to avoid the creation of many sub-lightpaths, as each one requires using guard bands which increases the wasted spectrum). On the other hand, the use of gridless instead of fixed-grid can also bring benefits as the fiber spectrum is used in a more efficient way in that scenario. When compared in terms of computing time, Joint Spectrum Gridless, the simplest of the four algorithms, is the one that requires the lowest computational time. In future works, the use of other RSA algorithms (instead of $k$-shortest-path and first fit) in combination with these two types of flexibility will be analyzed.

\section{REFERENCES}

[1] A. Mayoral et al., "Cascading of tenant SDN and cloud controllers for 5G network slicing using Transport API and Openstack API," Proc. OFC17, M2H.3, 2017.

[2] E. Wong et al., "Predictive Resource Allocation for Tactile Internet Capable Passive Optical LANs", Journal of Lightwave Technology, vol. 35, n.o 13, pp. 2629-2641, July 2017.

[3] B. Mukherjee, "Optical Communication Networks", McGraw-Hill, 1997.

[4] M. Jinno et al., "Spectrum-efficient and scalable elastic optical path network: Architecture, benefits, and enabling technologies," IEEE Communications Magazine, pp. 66-73, Nov. 2009.

[5] K. Christodoulopoulos et al., "Elastic bandwidth allocation in flexible OFDM-based optical networks", Journal of Lightwave Technology, vol. 29, no. 9, pp. 1354-1366, May 2011.

[6] K. Christodoulopoulos, I. Tomkos, E.A. Varvarigos, "Routing and spectrum allocation in OFDM-based optical networks with elastic bandwidth allocation," in Proc. IEEE GLOBECOM 2010.

[7] R.J. Durán et al., "Performance Comparison of Methods to Solve the Routing and Spectrum Allocation Problem," in Proc. ICTON 2012.

[8] I. Tomkos, S. Azodolmolky, J. Solé-Pareta, D. Careglio, and E. Palkopoulou, "A Tutorial on the Flexible Optical Networking Paradigm: State of the Art, Trends, and Research Challenges", Proceedings of the IEEE, vol. 102, no. 9, pp. 1317-1337, September 2014

[9] A. N. Patel et al., "Routing, wavelength assignment, and spectrum allocation algorithms in transparent flexible optical WDM networks," Optical Switching and Networking, vol. 9, no. 3, pp. 191-204, Jul. 2012.

[10] K. Klinkowski, M. Walkowiak, "Routing and spectrum assignment in spectrum sliced elastic optical path network," IEEE Communications Letters, vol.15, no. 8, pp. 884-886, Aug. 2011.

[11] D. Klonidis, F. Cugini, O. Gerstel, M. Jinno, V. López, E. Palkopoulou, M. Sekiya, D. Siracusa, G. Thouénon, and C. Betoule, "Spectrally and Spatially Flexible Optical Network Planning and Operations", IEEE Communications Magazine, vol. 53, no. 2, pp. 69-78, February 2015.

[12] H. Zang et al., "A review of routing and wavelength assignment approaches for wavelength-routed optical WDM networks," Optical Networks Magazine, pp. 47-60, Jan. 2000.

[13] OMNeT++ Discrete Event Simulator. https://omnetpp.org 